\title{
In Vitro Antioxidant Activity and Hepatoprotective Potential of Ceropegia spiralis Against Paracetamol Induced liver injury
}

\author{
Rajkiran Kolakota*, R. Santosh Kumar, Sunil Kumar Patnaik \\ Department of Pharmacology, Vignan Institute of Pharmaceutical Technology, Visakhapatnam, Andhra Pradesh, India.
}

\begin{tabular}{|c|c|}
\hline ARTICLE INFO & ABSTRACT \\
\hline Article history: & \multirow{12}{*}{$\begin{array}{l}\text { Ceropegia spiralis is a usually used remedial herb in herbal remedies to delight diverse diseases. The plan of the } \\
\text { current investigation is to assess the in vitro free radical scavenging activity and hepatoprotective activity of } \\
\text { Ceropegia spiralis against paracetamol induced liver damage in preventive and curative models. The Ceropegia } \\
\text { spiralis was evaluated for in vitro antioxidant activity by } 2,2 \text {-diphenyl-1-picryl hydrazyl (DPPH), hydroxyl and } \\
\text { superoxide radical scavenging activity and inhibition of lipid peroxide and ascorbic acid was used as a standard. } \\
\text { In two separate studies, the } 100,200 \text { and } 400 \mathrm{mg} / \mathrm{kg} \text { body weight of Ceropegia spiralis extract, and } 100 \mathrm{mg} / \mathrm{kg} \\
\text { body weight of Silymarin in both studies were given orally. The hepatic damage was done by oral } \\
\text { administration of } 2 \mathrm{~g} / \mathrm{kg} \text { body weight of paracetamol. The IC } 50 \text { values of Ceropegia spiralis in the superoxide, } 2 \text {, } \\
\text { 2-diphenyl-1-picryl hydrazyl (DPPH), hydroxyl radical scavenging activity and inhibition of lipid peroxidation } \\
\text { was found to be } 365.64,381.13,461.32 \text { and } 469.39 \mu \mathrm{g} / \mathrm{ml} \text { correspondingly. The hepatic damage in rats induced } \\
\text { by paracetamol as evidence by elevated serum aspartate aminotransferase (AST), alanine aminotransferase } \\
\text { (ALT), alkaline phosphatase (ALP) and total bilirubin levels and decreased serum total proteins. The } \\
\text { administration of Ceropegia spiralis and Silymarin in both preventive and curative models decreases the toxic } \\
\text { effect of paracetamol on the above selected serum parameters. }\end{array}$} \\
\hline Received on: $05 / 05 / 2017$ & \\
\hline Accepted on: 23/07/2017 & \\
\hline Available online: $30 / 09 / 2017$ & \\
\hline Key words: & \\
\hline Ceropegia spiralis, & \\
\hline Antioxidant, Paracetamol, & \\
\hline Hepatoprotective, Rat. & \\
\hline & \\
\hline & \\
\hline & \\
\hline & \\
\hline
\end{tabular}

\section{INTRODUCTION}

The liver has good impact in the regulation of homeostasis in the body. It maintains different biochemical pathways which are involved in the regulation of body growth, protect from certain disorders, provide nutritional support, production of energy and maintain reproductive consequences (Rajkiran et al., 2015; Nelson.1990 ).

The liver is the first target organ of xenobiotics which will alter the normal physiological functions and produce harmful effect. The liver protects the body from such type of unwanted toxic effects. The two main hepatotoxic events leads to mortality are Jaundice and hepatitis (Vermeulen et al., 1992).

\footnotetext{
* Corresponding Author

Dr. Rajkiran Kolakota, Associate Professor, Department of

Pharmacology, Vignan Institute of Pharmaceutical Technology, Visakhapatnam-530049, Andhra Pradesh, India.

Email: rajkiran.kolakota@gmail.com
}

Free radicals are the important inducing agents for the lipid peroxidations, cellular aging and diseases causing (Halliwell and Gutteridge, 1984). The Antioxidants are the substances which scavenge the free radicals like reactive oxygen species (ROS) and maintain the cytoskeleton of tissues (Barros et al., 2007; Sreenivasan et al., 2010). Nowadays the researchers are mainly focusing on the evaluation of various hepatoprotective agents for their paramount importance in the maintenance of the various metabolic activities and major protective site from different hazardous. A variety of literatures are indicating of a constant research on herbal hepatoprotective agents associated with antioxidant principle (Nithianantham et al., 2011; Soundararajan et al., 2012). Ceropegia spiralis (Famaly: Apocynaceae) is a small perennial herb, 20-30 cm tall, with weak, erect stem. Tubers are 1$2 \mathrm{~cm}$, spherical. Traditionally, this plant is used in the treatment of fever, indigestion and liver diseases (Yadav et al., 2006). The present investigation is to enumerate the hepatoprotective activity of methanolic extracts of Ceropegia spiralis, in higher dose of paracetamol induced hepatotoxicity. 


\section{MATERIALS AND METHODS}

Drug and chemicals

2-deoxy-D-ribose, Nitrobluetetrazolium (NBT) and 2, 2-diphenyl-1-picrylhydrazil (DPPH) were procured from SISCO Research laboratories Pvt Ltd, Mumbai and Sigma Chemical Co. (St. Louis, MO, USA) respectively. Paracetamol and Silymarin were obtained as a gift sample from Sri Krishna Pharmaceuticals, Mumbai, India and Micro Labs, Bangalore, India. The liver diagnostic kits like Aspartate aminotransferase (AST), alanine aminotransferase (ALT), alkaline phosphatase (ALP), Total bilirubin (TBR) and Total protein (TP) were procured from Agappe Diagnostics, Kerala, India. All additional chemicals and reagents used were of analytical grade.

\section{Preparation of methanolic extract}

The whole plant of Ceropegia spiralis were freshly collected and were subjected to shade dried. The dried whole plants were milled to coarse powder. One $\mathrm{kg}$ of the coarse powder and one liter of methanol $(95 \%)$ were taken in a round bottomed flask for the maceration process up to 24 hours at room temperature. The liquid extract was collected from soxhlet apparatus after successive extraction with 3 liter of methanol (95\%). The obtained liquid extract was subjected to rotary evaporator under reduced pressure until a soft mass obtained. The extracts were properly air dried to remove all traces of the solvent (Meharunnisha and Raj Kiran, 2016).

\section{In vitro antioxidant activity}

In present study inhibition of lipid peroxidation, Hydroxyl radical, Superoxide radical and DPPH radical scavenging activity were determined by Ohkawa et al., 1979, Elizabeth and Rao., 1990, Robak and Gryglewski et al., 1988 and Braca et al., 2003 respectively.

\section{Animals}

The male Albino Wistar rats $(180 \pm 200 \mathrm{~g})$ were selected for experimental work, which are procured from the Mahaveer Enterprises, Hyderabad, India. They were maintained in a constant environment temperature of $(23 \pm 2){ }^{\circ} \mathrm{C}$, humidity of $50 \%$ and 12 $\mathrm{h}: 12 \mathrm{~h}$ of light and dark cycles, respectively. All these animals were fed with the marketed pellet diet (Rayon's Biotechnology Pvt Ltd, India). The animals were access to water ad libitum. The experiment was supervised under the Institutional Animal Ethics Committee and is approved by CPCSEA, Government of India (Regd. No. 516/PO/C/01/ CPCSEA).

In vivo Hepatoprotective Study (Eswar Kumar et al., 2013; Rajkiran et al., 2015)

\section{Preventive study}

The male Albino Wistar rats were separated into six groups and each group has 6 rats.
Group A: Administered with 1\% sodium CMC for 3 days.

Group B: Administered with $2 \mathrm{~g} / \mathrm{kg}$ body weight of paracetamol for a period of 3 days.

Group C: Administered with $2 \mathrm{~g} / \mathrm{kg}$ body weight of paracetamol and Ceropegia spiralis extract $(100 \mathrm{mg} / \mathrm{kg}$ body weight) concurrently for 3 days.

Group D: Administered with $2 \mathrm{~g} / \mathrm{kg}$ body weight of paracetamol and Ceropegia spiralis extract $(200 \mathrm{mg} / \mathrm{kg}$ body weight) concurrently for 3 days.

Group E: Administered with $2 \mathrm{~g} / \mathrm{kg}$ body weight of paracetamol and Ceropegia spiralis extract $(400 \mathrm{mg} / \mathrm{kg}$ body weight) concurrently for 3 days.

Group F: Administered with $2 \mathrm{~g} / \mathrm{kg}$ body weight of paracetamol and Silymarin (100 mg/kg body weight) concurrently for 3 days.

Curative Study (Shenoy et al., 2002)

Group A: Control rats treated with $1 \%$ sodium CMC for 10 days.

Group B: Treated with paracetamol ( $2 \mathrm{~g} / \mathrm{kg}$ body weight) for first 3 days and then next seven days treated with $1 \%$ sodium CMC.

Group C: Treated with paracetamol ( $2 \mathrm{~g} / \mathrm{kg}$ body weight) for first 3 days and then next seven days treated with Ceropegia spiralis extract $(100 \mathrm{mg} / \mathrm{kg}$ body weight).

Group D: Treated with paracetamol (2 g/kg body weight) for first 3 days and then next seven days treated with Ceropegia spiralis extract $(200 \mathrm{mg} / \mathrm{kg}$ body weight).

Group E: Treated with paracetamol ( $2 \mathrm{~g} / \mathrm{kg}$ body weight) for first 3 days and then next seven days treated with Ceropegia spiralis extract $(400 \mathrm{mg} / \mathrm{kg}$ body weight).

Group F: Treated with paracetamol ( $2 \mathrm{~g} / \mathrm{kg}$ body weight) for first 3 days and then next seven days treated with Silymarin (100 mg/kg body weight).

All groups received paracetamol, Ceropegia spiralis and Silymarin orally. Silymarin was selected as standard agent for hepatoprotective activity. In preventive $\left(0^{\text {th }}\right.$ and $4^{\text {th }}$ day) and curative study $\left(0^{\text {th }}, 4^{\text {th }}\right.$ and $11^{\text {th }}$ day) blood samples were collected from rats retro-orbital plexus. The serum was isolated from blood by centrifugation at $3000 \mathrm{rpm}$ for $15 \mathrm{~min}$ for assessment of serum biochemical parameters.

The selected serum parameters like ALT, AST, ALP, serum Total bilirubin and Total protein estimated by Semi-auto analyzer (Agappe). After completion of the both studies the animals were sacrificed for isolation of livers for histopathological study and estimation of liver weight and volume. 


\section{Calculation of Percentage Hepatoprotection}

The following formula used for to calculate the percentage of liver protection produced by Ceropegia spiralis and Silymarin (Sintayehu et al., 2012).

$$
\mathrm{H}=\left(\frac{\mathrm{T}-\mathrm{B}}{\mathrm{T}-\mathrm{C}}\right) \times 100
$$

Where $\mathrm{T}=$ Group $\mathrm{B}$ mean value of marker.

B = Group C, Group D, Group E and Group F Mean value of marker.

$\mathrm{C}=$ Group A mean value of marker.

$\mathrm{H}=$ Percentage of liver protection.

\section{Statistical analysis}

The data was indicated as Mean \pm SEM produced from six animals. The Statistical analysis was done by using Prism-5 Graph Pad software. The one way analysis of variance (1 way ANOVA) with Bonferroni's multiple comparison test was used to compare the groups. The difference was less than 0.05 was considered to be statistically significant $(\mathrm{P}<0.05)$.

\section{RESULTS}

\section{In vitro antioxidant activity}

\section{Superoxide scavenging activity}

The Ceropegia spiralis and standard ascorbic acid showed concentration dependent scavenging activity on superoxide radical at different concentrations $(10-1000 \mu \mathrm{g})$.
The $C$. spiralis needed $365.64 \mu \mathrm{g}$ for $50 \%$ scavenging of superoxide radicals; whereas standard ascorbic acid needed 156.50 $\mu \mathrm{g}$ (Table 1 \& Histogram 1).

\section{Lipid peroxidation inhibiting activity}

The Ceropegia spiralis and standard ascorbic acid showed concentration dependent inhibition of lipid peroxidation at different concentrations $(10-1000 \mu \mathrm{g})$. The $C$. spiralis needed $469.39 \mu \mathrm{g}$ for $50 \%$ inhibition of lipid peroxidation; whereas standard ascorbic acid needed $125.69 \mu \mathrm{g}$ (Table $1 \&$ Histogram 1).

\section{Hydroxyl radical scavenging activity}

The Ceropegia spiralis and standard ascorbic acid showed concentration dependent scavenging activity on hydroxyl radical at different concentrations (10-1000 $\mu \mathrm{g})$. The $C$. spiralis needed $461.32 \mu \mathrm{g}$ for $50 \%$ scavenging of hydroxyl radicals; whereas standard ascorbic acid needed $237.49 \mu \mathrm{g}$ (Table 1 \& Histogram 1).

\section{DPPH radical scavenging activity}

The Ceropegia spiralis and standard ascorbic acid showed concentration dependent scavenging activity on DPPH radical at different concentrations $(10-1000 \mu \mathrm{g})$. The $C$. spiralis needed $381.13 \mu \mathrm{g}$ for $50 \%$ scavenging of DPPH radicals; whereas standard ascorbic acid needed $100.13 \mu \mathrm{g}$ (Table $1 \&$ Histogram1).

Table 1: In vitro antioxidant activity of methanolic extract of Ceropegia spiralis \& Ascorbic acid.

\begin{tabular}{|c|c|c|c|c|c|c|c|c|}
\hline \multirow{3}{*}{$\begin{array}{l}\text { Conc. } \\
(\mu \mathrm{g} / \mathrm{ml})\end{array}$} & \multicolumn{8}{|c|}{ Percentage Inhibition of free radicals } \\
\hline & \multicolumn{2}{|c|}{ Super oxide radical } & \multicolumn{2}{|c|}{ Hydroxyl radical } & \multicolumn{2}{|c|}{ Lipid peroxidation } & \multicolumn{2}{|c|}{ DPPH radical } \\
\hline & Ascorbic acid & Ceropegia spiralis & Ascorbic acid & $\begin{array}{c}\text { Ceropegia } \\
\text { spiralis }\end{array}$ & $\begin{array}{c}\begin{array}{c}\text { Ascorbic } \\
\text { acid }\end{array} \\
\end{array}$ & $\begin{array}{c}\text { Ceropegia } \\
\text { spiralis }\end{array}$ & $\begin{array}{c}\begin{array}{c}\text { Ascorbic } \\
\text { acid }\end{array} \\
\end{array}$ & Ceropegia spiralis \\
\hline 10 & $25.68 \pm 0.22$ & $15.26 \pm 0.62$ & $19.56 \pm 0.47$ & $20.23 \pm 4.05$ & $31.51 \pm 0.07$ & $24.26 \pm 0.09$ & $24.72 \pm 0.84$ & $18.20 \pm 1.81$ \\
\hline 25 & $34.58 \pm 0.08$ & $24.11 \pm 0.36$ & $28.26 \pm 1.01$ & $26.55 \pm 4.25$ & $34.73 \pm 0.07$ & $30.34 \pm 0.09$ & $35.06 \pm 1.79$ & $29.85 \pm 0.06$ \\
\hline 50 & $47.48 \pm 0.44$ & $37.36 \pm 0.64$ & $36.98 \pm 0.63$ & $35.65 \pm 4.14$ & $48.23 \pm 0.08$ & $36.50 \pm 0.06$ & $49.91 \pm 0.45$ & $36.83 \pm 0.04$ \\
\hline 100 & $59.44 \pm 0.66$ & $46.76 \pm 0.06$ & $54.46 \pm 0.87$ & $47.10 \pm 2.41$ & $60.18 \pm 0.10$ & $39.73 \pm 0.05$ & $63.83 \pm 0.36$ & $47.58 \pm 0.03$ \\
\hline 250 & $66.62 \pm 4.24$ & $55.39 \pm 0.04$ & $77.99 \pm 0.66$ & $57.68 \pm 5.15$ & $66.36 \pm 0.05$ & $51.92 \pm 0.03$ & $72.58 \pm 0.07$ & $54.77 \pm 0.06$ \\
\hline 500 & $73.47 \pm 0.09$ & $66.30 \pm 0.90$ & $80.65 \pm 0.57$ & $49.68 \pm 6.94$ & $74.56 \pm 0.09$ & $59.21 \pm 0.03$ & $75.26 \pm 0.08$ & $62.62 \pm 0.05$ \\
\hline 1000 & $83.49 \pm 0.10$ & $73.52 \pm 0.05$ & $82.63 \pm 0.55$ & $61.81 \pm 2.18$ & $78.92 \pm 0.03$ & $61.65 \pm 0.07$ & $85.52 \pm 0.10$ & $68.20 \pm 0.04$ \\
\hline $\mathrm{IC}_{50}$ & 156.30 & 365.64 & 237.49 & 461.32 & 125.69 & 469.39 & 100.13 & 381.13 \\
\hline
\end{tabular}

Values are mean \pm S.D three replicates.

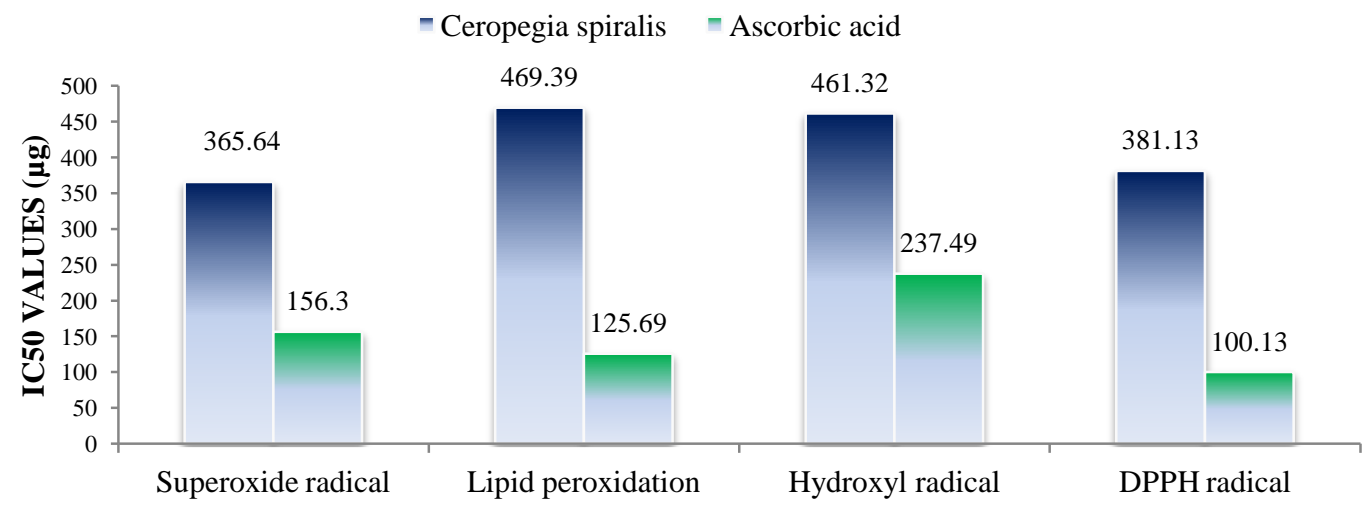

Histogram 1: In vitro $50 \%$ inhibition concentration $\left(\mathrm{IC}_{50}\right)$ of methanolic extracts of $C$. spiralis and ascorbic acid on free radicals scavenging activity. 
Table 2: Effect of methanolic extract of Ceropegia spiralis and Silymarin on serum biochemical parameters on paracetamol induced hepatotoxicity in rats (preventive study).

\begin{tabular}{|c|c|c|c|c|c|c|c|c|c|c|}
\hline Group/ & \multicolumn{2}{|c|}{ SGPT(IU/L) } & \multicolumn{2}{|c|}{ SGOT(IU/L) } & \multicolumn{2}{|c|}{ ALP(IU/L) } & \multicolumn{2}{|c|}{ T.BIL(mg/dl) } & \multicolumn{2}{|c|}{ T.PR(g/dl) } \\
\hline Treatment & $0^{\text {th }}$ Day & $4^{\text {th }}$ Day & $0^{\text {th }}$ Day & $4^{\text {th }}$ Day & $0^{\text {th }}$ Day & $4^{\text {th }}$ Day & $0^{\text {th }}$ Day & $4^{\text {th }}$ Day & $0^{\text {th }}$ Day & $4^{\text {th }}$ Day \\
\hline Gro & $28 \pm 3.47$ & $59.35 \pm 2.6$ & $2 \pm 3$ & $83.78 \pm 2.6$ & $.25 \pm 2.71$ & $144.98 \pm 2$ & $1 \pm 0.02$ & 0.3 & $57 \pm 0.22$ & 25 \\
\hline & $38 \pm 3.71$ & $3.73=$ & 31 & $9.18 \pm 8$ & $44 \pm 4.85$ & $8.35 \pm 3$. & $6 \pm 0.03$ & $5.71 \pm 0.31^{\# \# \#}$ & $97 \pm 0.35$ & $3.94 \pm 0.22^{\# \# \#}$ \\
\hline Gro & $73 \pm 2.46$ & $202.68 \pm 4$ & $91.22 \pm 3.45$ & $304.04 \pm 5$. & $9.65 \pm 2.16$ & $343.48 \pm 4$. & $0.38 \pm 0.04$ & $5.06 \pm 0.24^{\mathrm{ns}}$ & $7.93 \pm 0.44$ & $4.02 \pm 0.16^{\mathrm{ns}}$ \\
\hline Grou & $.66 \pm 3.97$ & $50.12 \pm 5$ & $.42 \pm 2.98$ & $55.49 \pm 7.3$ & $8.61 \pm 4.94$ & 16.2 & $9 \pm 0.04$ & $4.36 \pm 0.25^{*}$ & $7.91 \pm 0.45$ & $4.37 \pm 0.31^{\mathrm{ns}}$ \\
\hline Group E & $56.63 \pm 3.41$ & $133.23 \pm 3.69 * * *$ & $89.08 \pm 4.17$ & $228.33 \pm 6.62 * * *$ & $140.51 \pm 4.60$ & $284.59 \pm 5.1$ & $.35 \pm 0.04$ & $3.90 \pm 0.20 * * *$ & $8.02 \pm 0.30$ & $4.94 \pm 0.29^{\mathrm{ns}}$ \\
\hline Group F & $58.36 \pm 3.41$ & $65.79 \pm 4.69 * * *$ & $88.19 \pm 2.95$ & $101.46 \pm 5.18^{* * *}$ & $152.46 \pm 3.71$ & $181.25 \pm 5.29 * * *$ & $0.39 \pm 0.03$ & $0.45 \pm 0.11 * * *$ & $7.07 \pm 0.34$ & $6.78 \pm 0.23 * * *$ \\
\hline
\end{tabular}

Values are the Mean \pm S.E.M. of six rats for each treatment; ${ }^{\# \#}$ Significance $\mathrm{P}<0.001$ when Group B compared with Group A; *** Significance P <0.001, ** $\mathrm{P}<0.01,{ }^{*} \mathrm{P}<0.05$ when rest of Groups compared with PCM treated group (Group B). NOTE: Group A: Normal 1\%Sod.CMC, Group B: Treated with Paracetamol $(2 \mathrm{~g} / \mathrm{kg})$, Group C: Treated with Paracetamol(2g/kg) + MECS (100mg/kg), Group D: Treated with Paracetamol $(2 \mathrm{~g} / \mathrm{kg})+\mathrm{MECS}(200 \mathrm{mg} / \mathrm{kg})$, Group E: Treated with Paracetamol $(2 \mathrm{~g} / \mathrm{kg})+$ MECS $(400 \mathrm{mg} / \mathrm{kg})$ and Group F: Treated with Paracetamol $(2 \mathrm{~g} / \mathrm{kg})+$ Silymarin $100 \mathrm{mg} / \mathrm{kg})$.

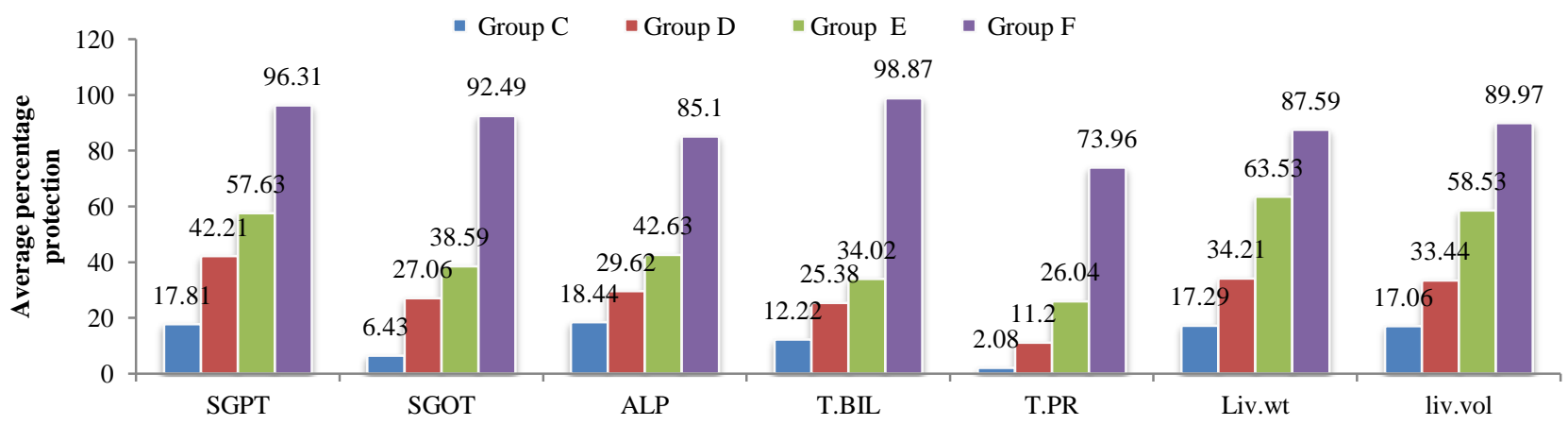

Histogram 2: Average percentage protection produced by methanolic extract of Ceropegia spiralis and Silymarin against PCM induced hepatotoxicity (preventive study). NOTE: Group C: Treated with Paracetamol $(2 \mathrm{~g} / \mathrm{kg})+$ MECS $(100 \mathrm{mg} / \mathrm{kg})$, Group D: Treated with Paracetamol $(2 \mathrm{~g} / \mathrm{kg})+\mathrm{MECS}$ $(200 \mathrm{mg} / \mathrm{kg})$, Group E: Treated with Paracetamol $(2 \mathrm{~g} / \mathrm{kg})+$ MECS $(400 \mathrm{mg} / \mathrm{kg})$ and Group F: Treated with Paracetamol $(2 \mathrm{~g} / \mathrm{kg})+\mathrm{Silymarin} 100 \mathrm{mg} / \mathrm{kg})$.

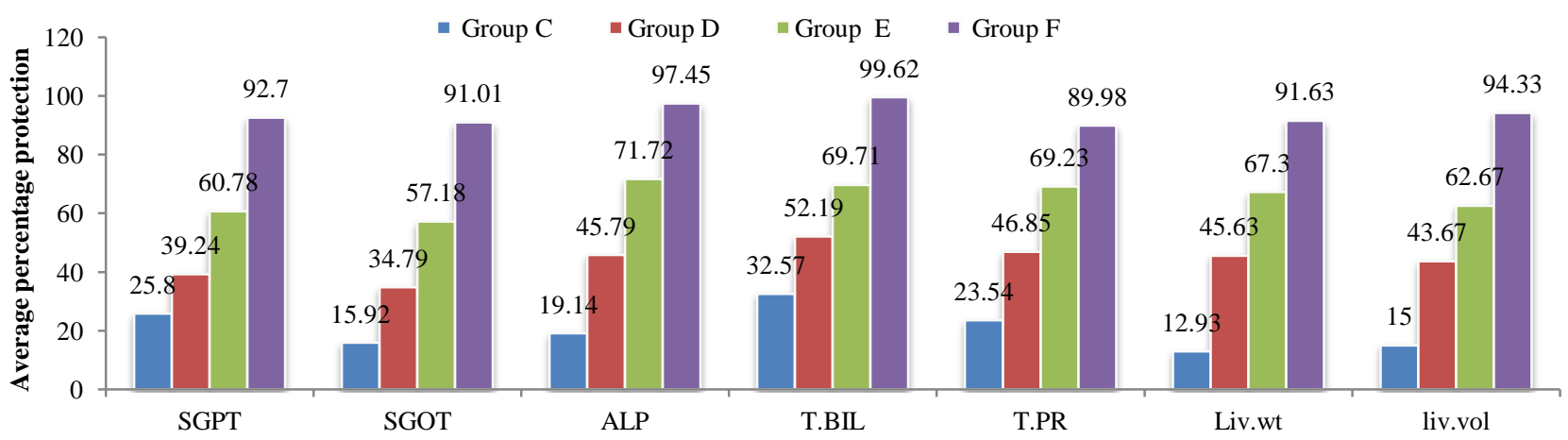

Histogram 3: Average percentage protection produced by methanolic extract of Ceropegia spiralis and Silymarin against PCM induced hepatotoxicity (curative study). NOTE: Group C: Treated with Paracetamol (2g/kg) + MECS (100mg/kg), Group D: Treated with Paracetamol (2g/kg) + MECS (200mg/kg), Group E: Treated with Paracetamol $(2 \mathrm{~g} / \mathrm{kg})+$ MECS $(400 \mathrm{mg} / \mathrm{kg})$ and Group F: Treated with Paracetamol $(2 \mathrm{~g} / \mathrm{kg})+$ Silymarin $100 \mathrm{mg} / \mathrm{kg})$.

\section{In vivo Hepatoprotective Activity}

\section{Estimation of Serum Biochemical Parameters}

The paracetamol treated group (Group-B) showed significant $(\mathrm{p}<0.01)$ increase in selected serum biochemical parameters namely AST, ALT, ALP and total bilirubin and serum levels of total protein significantly decreased when compared with normal group. However, the administration of Ceropegia spiralis at different doses and Silymarin showed significant $(\mathrm{p}<0.01)$ reduction in serum biochemical parameters like AST, ALT, ALP and total bilirubin levels and serum levels of total protein significantly elevated when compared to paracetamol treated group in both preventive and curative study (Table 2 to 4 ).

The results of the study based on levels of AST, ALT, ALP, T. BIL and T. PR biochemical parameters the rats treated with $400 \mathrm{mg} / \mathrm{kg}$ body of Ceropegia spiralis produced better recovery $57.63 \%, 38.59 \%, 42.63 \%, 34.02 \%$ and $26.04 \%$ against paracetamol intoxication respectively in preventive study and $60.78 \%, 57.18 \%, 71.72 \%, 69.71 \%$ and $69.23 \%$ respectively in curative study (Histogram $2 \&$ Histogram 3). 


\section{Estimation of physical parameters}

In our study the paracetamol treated group showed significant $(\mathrm{p}<0.01)$ increase in levels of physical parameters like liver weight and volume when compared with normal rats. However, the rats treated with different doses of Ceropegia spiralis and Silymarin showed significant $(\mathrm{p}<0.01)$ reduction in physical parameters like liver weight and volume when compared to paracetamol treated group in both preventive and curative study (Table 5).

The based on the liver weight and volume the $400 \mathrm{mg} / \mathrm{kg}$ body weight of Ceropegia spiralis produced better recovery $63.53 \%$ and $58.53 \%$ against paracetamol intoxication respectively in preventive study and $67.30 \%$ and $62.67 \%$ respectively in curative study (Histogram 2 \& Histogram 3).

Table 3: Effect of methanolic extract of Ceropegia spiralis and Silymarin on serum biochemical parameters on paracetamol induced hepatotoxicity in rats (curative study).

\begin{tabular}{|c|c|c|c|c|c|c|c|c|c|}
\hline \multirow{2}{*}{$\begin{array}{c}\text { Group/ } \\
\text { Treatment }\end{array}$} & \multicolumn{3}{|c|}{ SGPT(IU/L) } & \multicolumn{3}{|c|}{ SGOT(IU/L) } & \multicolumn{3}{|c|}{ ALP(IU/L) } \\
\hline & $0^{\text {th }}$ Day & $4^{\text {th }}$ Day & $11^{\text {th }}$ Day & $0^{\text {th }}$ Day & $4^{\text {th }}$ Day & $11^{\text {th }}$ Day & $0^{\text {th }}$ Day & $4^{\text {th }}$ Day & $11^{\text {th }}$ Day \\
\hline Group A & $62.43 \pm 1.94$ & $58.15 \pm 1.95$ & $60.19 \pm 2.80$ & $83.52 \pm 1.77$ & $85.26 \pm 3.31$ & $85.29 \pm 2.42$ & $140.17 \pm 3.42$ & $140.89 \pm 2.06$ & $137.54 \pm 2.17$ \\
\hline Group B & $57.57 \pm 2.64$ & $221.32 \pm 3.75$ & $210.25 \pm 2.91^{\# \# \# ~}$ & $86.20 \pm 3.94$ & $320.71 \pm 5.85$ & $311.17 \pm 6.36^{\# \# \#}$ & $151.48 \pm 3.83$ & $396.91 \pm 6.85$ & $372.33 \pm 7.52^{\# \# \# ~}$ \\
\hline Group C & $65.91 \pm 1.67$ & $227.06 \pm 3.52$ & $171.54 \pm 3.33 * * *$ & $81.22 \pm 2.68$ & $339.46 \pm 3.55$ & $275.22 \pm 5.35^{* * * *}$ & $154.80 \pm 6.48$ & $410.23 \pm 3.54$ & $327.39 \pm 3.91 * * *$ \\
\hline Group D & $60.76 \pm 2.58$ & $220.73 \pm 6.14$ & $151.37 \pm 3.67 * * *$ & $83.65 \pm 1.93$ & $329.11 \pm 6.91$ & $232.59 \pm 5.48 * * *$ & $160.98 \pm 2.74$ & $408.60 \pm 5.56$ & $264.81 \pm 7.52 * * *$ \\
\hline Group E & $59.54 \pm 0.66$ & $227.66 \pm 5.52$ & $119.04 \pm 5.77 * * *$ & $84.20 \pm 1.59$ & $321.21 \pm 4.39$ & $182.02 \pm 4.98 * * *$ & $141.96 \pm 3.14$ & $426.48 \pm 5.20$ & $203.95 \pm 6.21 * * *$ \\
\hline Group F & $59.83 \pm 2.72$ & $234.06 \pm 3.64$ & $71.14 \pm 3.43 * * *$ & $94.93 \pm 2.09$ & $338.93 \pm 3.64$ & $105.59 \pm 3.30 * * *$ & $142.51 \pm 2.74$ & $408.31 \pm 2.15$ & $143.53 \pm 3.30 * * *$ \\
\hline
\end{tabular}

Values are the Mean \pm S.E.M. of six rats for each treatment; ${ }^{\# \#}$ Significance $\mathrm{P}<0.001$ when Group B compared with Group A; $* * *$ Significance P <0.001, **P $<0.01,{ }^{*} \mathrm{P}<0.05$ when rest of Groups compared with PCM treated group (Group B). NOTE: Group A: Normal 1\%Sod.CMC, Group B: Treated with Paracetamol(2g/kg), Group C: Treated with Paracetamol(2g/kg) + MECS (100mg/kg), Group D: Treated with Paracetamol(2g/kg) + MECS (200mg/kg), Group E: Treated with Paracetamol $(2 \mathrm{~g} / \mathrm{kg})+\operatorname{MECS}(400 \mathrm{mg} / \mathrm{kg})$ and Group F: Treated with Paracetamol $(2 \mathrm{~g} / \mathrm{kg})+\mathrm{Silymarin} 100 \mathrm{mg} / \mathrm{kg})$.

Table 4: Effect of methanolic extract of Ceropegia spiralis and Silymarin on serum biochemical parameters on paracetamol induced hepatotoxicity in rats (curative study).

\begin{tabular}{|c|c|c|c|c|c|c|}
\hline \multirow{2}{*}{$\begin{array}{c}\text { Group/ } \\
\text { Treatment }\end{array}$} & \multicolumn{3}{|c|}{ T.BIL(mg/dl) } & \multicolumn{3}{|c|}{ T.PR(g/dl) } \\
\hline & $0^{\text {th }}$ Day & $4^{\text {th }}$ Day & $11^{\text {th }}$ Day & $0^{\text {th }}$ Day & $4^{\text {th }}$ Day & $11^{\text {th }}$ Day \\
\hline Group A & $0.34 \pm 0.04$ & $0.43 \pm 0.07$ & $0.37 \pm 0.03$ & $8.04 \pm 0.38$ & $8.01 \pm 0.32$ & $8.13 \pm 0.43$ \\
\hline Group B & $0.32 \pm 0.13$ & $5.78 \pm 0.32$ & $5.69 \pm 0.37^{\# \# \#}$ & $8.47 \pm 0.35$ & $3.72 \pm 0.17$ & $3.84 \pm 0.37^{\# \# \#}$ \\
\hline Group C & $0.27 \pm 0.05$ & $5.90 \pm 0.41$ & $3.98 \pm 0.32 * * *$ & $7.32 \pm 0.27$ & $4.19 \pm 0.18$ & $4.85 \pm 0.24^{\mathrm{ns}}$ \\
\hline Group D & $0.38 \pm 0.07$ & $6.16 \pm 0.38$ & $2.95 \pm 0.13 * * *$ & $8.84 \pm 0.46$ & $3.86 \pm 0.31$ & $5.85 \pm 0.30 * * *$ \\
\hline Group E & $0.35 \pm 0.07$ & $6.69 \pm 0.31$ & $2.03 \pm 0.32 * * *$ & $8.02 \pm 0.38$ & $3.92 \pm 0.34$ & $6.81 \pm 0.23 * * *$ \\
\hline Group F & $0.38 \pm 0.05$ & $6.04 \pm 0.30$ & $0.46 \pm 0.10 * * *$ & $8.00 \pm 0.35$ & $4.50 \pm 0.19$ & $7.70 \pm 0.13 * * *$ \\
\hline
\end{tabular}

${ }^{\mathrm{a}}$ Values are the Mean \pm S.E.M. of six rats for each treatment; ${ }^{\# \# \#}$ Significance $\mathrm{P}<0.001$ when Group B compared with Group A; $* * *$ Significance P $<0.001$, $* * \mathrm{P}<0.01,{ }^{*} \mathrm{P}<0.05$ when rest of Groups compared with PCM treated group(Group B). NOTE: Group A: Normal 1\% Sod.CMC, Group B: Treated with Paracetamol $(2 \mathrm{~g} / \mathrm{kg})$, Group C: Treated with Paracetamol $(2 \mathrm{~g} / \mathrm{kg})+$ MECS $(100 \mathrm{mg} / \mathrm{kg})$, Group D: Treated with Paracetamol $(2 \mathrm{~g} / \mathrm{kg})+\mathrm{MECS}(200 \mathrm{mg} / \mathrm{kg}), \mathrm{Group}$ E: Treated with Paracetamol $(2 \mathrm{~g} / \mathrm{kg})+$ MECS $(400 \mathrm{mg} / \mathrm{kg})$ and Group F: Treated with Paracetamol $(2 \mathrm{~g} / \mathrm{kg})+\mathrm{Silymarin} 100 \mathrm{mg} / \mathrm{kg})$.

Table 5: Effect of methanolic extract of Ceropegia spiralis and Silymarin on physical parameters on paracetamol induced hepatotoxicity in rats.

\begin{tabular}{|c|c|c|c|c|}
\hline \multirow{2}{*}{ Group/ Treatment } & \multicolumn{2}{|c|}{ Preventive study } & \multicolumn{2}{|c|}{ Curative study } \\
\hline & Liv.wt ( g/100g ) & Liv.vol (ml/100g ) & Liv.wt ( g/100g ) & Liv.vol (ml/100g ) \\
\hline Group A & $3.24 \pm 0.06$ & $3.57 \pm 0.07$ & $3.38 \pm 0.08$ & $3.71 \pm 0.07$ \\
\hline Group B & $5.90 \pm 0.19^{\# \# \#}$ & $6.56 \pm 0.21^{\# \# \#}$ & $6.01 \pm 0.12^{\# \# \#}$ & $6.71 \pm 0.16^{\# \# \#}$ \\
\hline Group C & $5.44 \pm 0.14^{\mathrm{ns}}$ & $6.05 \pm 0.16^{\mathrm{ns}}$ & $5.67 \pm 0.08^{\mathrm{ns}}$ & $6.26 \pm 0.13^{\mathrm{ns}}$ \\
\hline Group D & $4.99 \pm 0.18 * *$ & $5.56 \pm 0.19 * *$ & $4.81 \pm 0.13^{* * *}$ & $5.40 \pm 0.19 * * *$ \\
\hline Group E & $4.21 \pm 0.11 * * *$ & $4.81 \pm 0.14^{* * *} *$ & $4.24 \pm 0.12 * * *$ & $4.83 \pm 0.12 * * *$ \\
\hline Group F & $3.57 \pm 0.09 * * *$ & $3.87 \pm 0.10^{* * *}$ & $3.60 \pm 0.13^{* * *}$ & $3.88 \pm 0.15^{* * *}$ \\
\hline
\end{tabular}



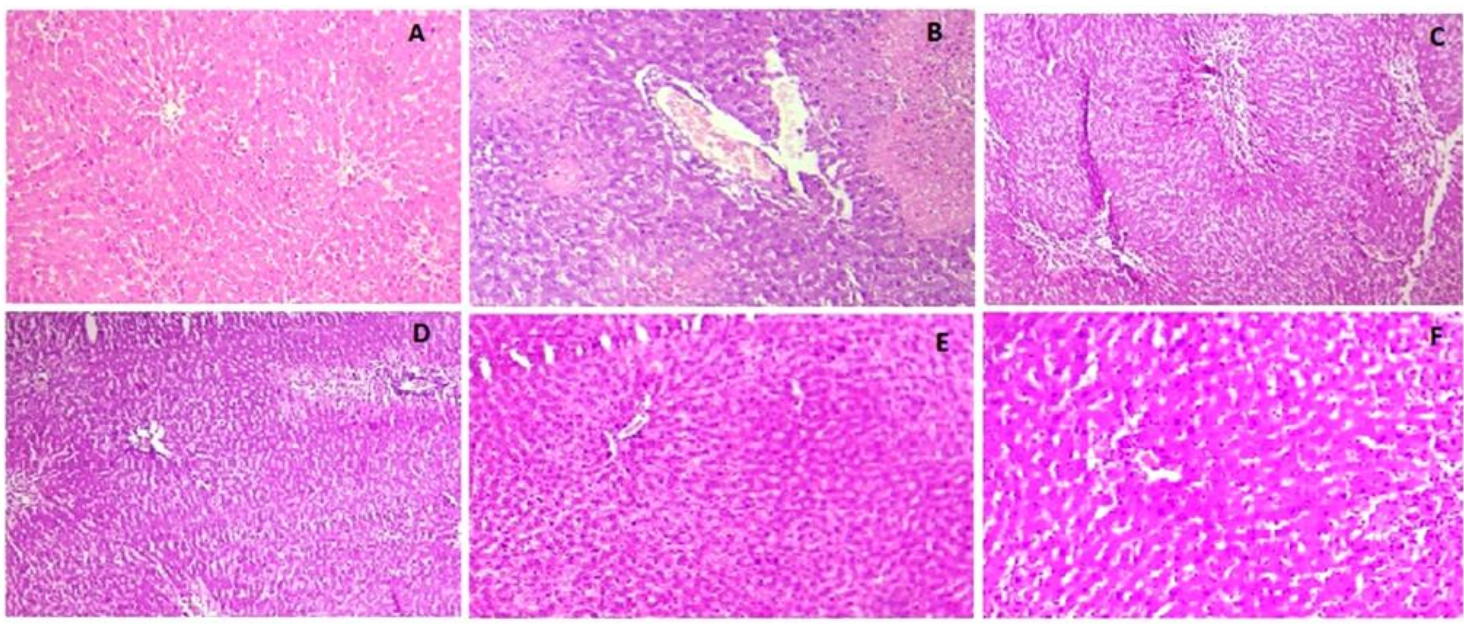

Figure No.1: Histopathology of liver tissues in control and experimental groups of rats (Preventive study). (A) Normal control group: showed the well defined architecture and vesicular nuclei. (B) Hepatotoxic group: showed degenerative changes in hepatocytes and necrosis occurred. (C) Group treated with MECS $100 \mathrm{mg} / \mathrm{kg}$ : showed proliferative hepatocytes no degenerative changes and increased inter cellular space. (D) Group treated with MECS $200 \mathrm{mg} / \mathrm{kg}$ : showed proliferative hepatocytes no degenerative changes and increased inter cellular space (E) Group treated with MECS $400 \mathrm{mg} / \mathrm{kg}$ : showed better architecture and well defined nuclei when compared with group A. (F) Group treated with Silymarin $100 \mathrm{mg} / \mathrm{kg}$ : section showed normal architecture with well defined nucleus.
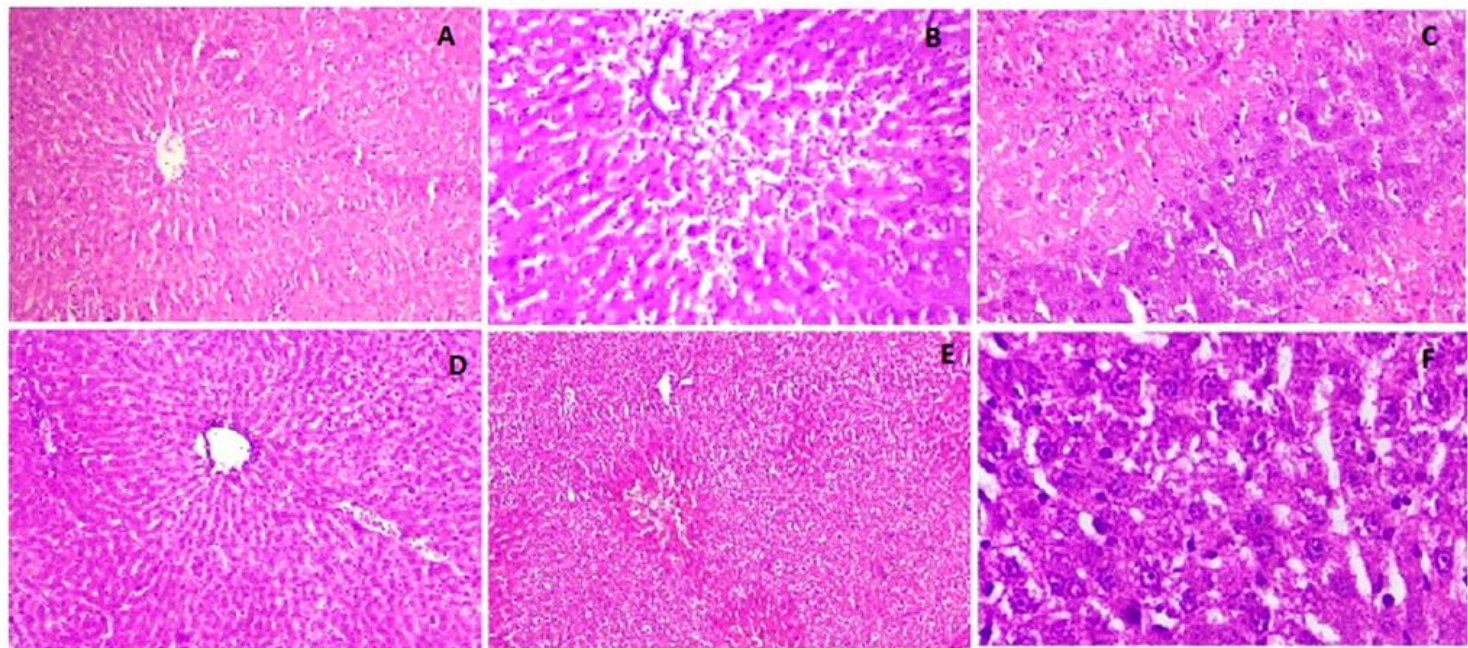

Figure No.2: Histopathology of liver tissues in control and experimental groups of rats (Curative study). (A) Normal control group: showed the well defined architecture and vesicular nuclei. (B) Hepatotoxic group: showed degenerative changes in hepatocytes and necrosis occurred. (C) Group treated with MECS $100 \mathrm{mg} / \mathrm{kg}$ : showed proliferative hepatocytes no degenerative changes and increased inter cellular space. (D) Group treated with MECS $200 \mathrm{mg} / \mathrm{kg}$ : showed proliferative hepatocytes mild degenerative changes and increased inter cellular space (E) Group treated with MECS $400 \mathrm{mg} / \mathrm{kg}$ : showed clear cells, nucleated, follows normal structure and architecture with sign of very mild inflammation when compared with group A. (F) Group treated with Silymarin $100 \mathrm{mg} / \mathrm{kg}$ : section showed normal architecture with well defined nucleus.

\section{Histopathological studies}

The histopathological study of liver was done as a supportive parameter for the hepatoprotective action shown by the methanolic extract of Ceropegia spiralis in both preventive and curative studies. The normal architecture of hepatocytes contains number of hexagonal/polygonal lobules, with more eosinophilic cytoplasm (Naidu et al., 2007). The same features were observed in normal control group (Fig. no. $1 \& 2$ ).
The histopathological study of paracetamol treated group (Group B) in both preventive as well as curative studies shown a high derangement of hepatic cords, ballooning, necrosis, cellular infiltration and loss of cell boundaries without any sign of regeneration (Fig. no. 1 \& 2), whereas Ceropegia spiralis treated groups showed decrease in these abnormalities in dose dependent manner with poor regeneration (Fig. no. $1 \& 2$ ). 


\section{DISCUSSION}

A low dose of Paracetamol is widely used as antipyretic and analgesic agent but a higher dose can produces hepatic damage in humans as well as in rodents. It is a well adapted model for screening of hepatoprotective agents. The N-acetyl-pbenzoquinoneimine (NAPQI) is the metabolic product of Paracetamol, which trigger the hepatotoxicity (Lee et al., 1991) by generating the reactive oxygen species (ROS). Consequently oxidative stress is overwhelming and leads to hepatic injury and hepatic death (McGill et al., 2012; Yanpallewar et al., 2002). The enzymes present in cytoplasm are rushed into the systemic circulation in order to damage of the liver cell membrane, (Ramaiah. 2007). Hence, the elevated serum cytosolic enzymes level indicates the quantitative biomarkers of hepatic damage.

The laboratory findings of paracetamol (PCM) induced hepatotoxicity are similar as other acute hepatic inflammation and enhancement of liver ailment with major increase of AST, ALP, ALT, LDH, cholesterol, bilirubin and decrease of TP (Davidson and Eastham, 1996). The experimental data clearly referred the elevation of serum hepatic enzymes level such as AST, ALP, ALT, total bilirubin, and reduce level of Total Protein, which conveying the hepatic damage in the PCM induced hepatotoxic rats. The Methanolic extract of Ceropegia spiralis at various doses of 100,200 and $400 \mathrm{mg} / \mathrm{kg}$ significantly ( $\mathrm{p}<0.05$ to $\mathrm{p}<0.01$ ) lowered the AST, ALP, ALT, total bilirubin and physical parameters, and total protein levels were elevated in these PCM intoxicated rats and also offered maximum recovery at a dose of $400 \mathrm{mg} / \mathrm{kg}$ body weight in both studies.

The data obtained from the liver function tests were analyzed with histopathological changes from photomicrographs taken. The centrilobular hepatic necrosis, cell degeneration and infiltrating lymphocytes were well marked in PCM intoxicated group. Treatment with methanolic extract of Ceropegia spiralis prevented these PCM induced histopathological changes. These results of the study showed inhibition of hepatic damage and elevated hepatic function markers may participate in the protective effect of the methanolic extract of Ceropegia spiralis against paracetamol induced hepatotoxicity in both preventive and curative studies.

The results further indicates among these two studies the methanolic extract of Ceropegia spiralis showed better results in curative study against paracetamol induced hepatotoxicity. The hepatoprotective activity of methanolic extract of Ceropegia spiralis might be due to its free radical scavenging property, which reduce the oxidative stress imposed by paracetamol.

\section{CONCLUSION}

The present investigation concludes that, the methanolic extract of Ceropegia spiralis have hepatoprotective activity in preventive and curative study, which is due to its antioxidant activity. A further investigation has to carry out for the identification of active principle responsible for hepatoprotective action.

\section{ACKNOWLEDGEMENTS}

The authors are grateful to Dr. K. Eswar Kumar, Associate professor, Department of Pharmacology, A.U college of Pharmaceutical Sciences, Andhra University, India for providing laboratory facilities for extraction of the plant material and its evaluation for pharmacological activities respectively.

\section{Financial support and sponsorship: Nil.}

Conflict of Interests: There are no conflicts of interest.

\section{REFERENCES}

Barros, L.; Baptista, P. Ferreira ICFR: Effect of Lactarius piperatus fruiting body maturity stage on antioxidant activity measured by several biochemical assays. Food. Chem. Toxicol. 2007; 45: 1731-1737.

Biruk Sintayehu1., Franz Bucar., Ciddi Veeresham and Kaleab Asres1. Hepatoprotective and free radical scavenging activities of extracts and a major compound isolated from the leaves of Cineraria abyssinica Sch. Bip. exA. Rich. Pharmacognocy journal. 2012; 4(29):4046.

Braca A, Fico G, Morelli I, De Simone F, Tome F and De Tommasi N. Antioxidant and free radical scavenging activity of flavonol glycosides from different Aconitum species. Journal of Ethnopharmacology. 2003; 86(1):63-67.

Davidson DG and Eastham WN. Acute liver necrosis following overdose of paracetamol. Br Med J. 1966; 2:497e499.

Elizabeth $\mathrm{K}$ and Rao MNA. Oxygen radical scavenging activity of curcumin. International Journal of Pharmaceutics. 1990; 58(3):237-240

Eswar Kumar K., Harsha KN., Neelakanta Rao N., Sudheer V., and Giri Babu N. In vitro antioxidant activity and in vivo hepatoprotective activity of aqueous extract of Allium cepa bulb in paracetamol induced liver damage in Wistar rats. Research Journal of Pharmaceutical, Biological and Chemical Sciences. 2013; 4(2): 338

Halliwell B and Gutteridge J.M.C. Oxygen toxicity, oxygen radicals, transition metals and disease. Biochem. J. 1984; 219: 1-4

K. Nithianantham., M. Shyamala., Y. Chen., L.Y. Latha., S.L. Jothy., and S. Sasidharan. "Hepatoprotective Potential of Clitoria ternatea Leaf Extract Against Paracetamol Induced Damage in Mice," Molecules. 2011;16: 10134-10145.

Lee CA., Thummel KE., Kalhorn TF., Nelson SD and Slattery JT. Drug Metab Dispos. 1991; 19(5): 966-971.

McGill MR., Williams CD., Xie Y., Ramachandran A and Jaeschke H. Toxicol Appl Pharmacol, 2012; 264(3): 387-394.

Mohmed Meharunnisha Begum and K. Raj Kiran. Evaluation of Methanolic Extract of Cleome chelidonii for Hepatoprotective Activity against Paracetamol and Ethanol Induced Hepatotoxicity in Rats. International Journal of Pharma Research \& Review. 2016; 5(1):28-36.

Nelson SD. Molecular mechanisms of the hepatotoxicity caused by acetaminophen. Seminliver dis. 1990; 10: 267-268

Ohkawa H., Ohishi N and Yagi K. Assay for lipid peroxides in animal tissues by thiobarbituric acid reaction. Analytical Biochemistry. 1979; 95(2):351-358.

Rajkiran K., Eswar Kumar K., Satyanarayana S. Evaluation of in vitro antioxidant activity of aqueous extract of root of Coleus edulis and its correlation with in vivo hepatoprotective activity in paracetamol induced hepatotoxicity in rats. Asian J Pharm Clin Res. 2015; 8(4): 56-61

Ramaiah SK. A toxicologist guide to the diagnostic interpretation of hepatic biochemical parameters. Food Chem. Toxicol. 2007; 45(4): 1551-1557.

Robak J and Gryglewski RJ. Flavonoids are scavengers of superoxide anions. Biochemical Pharmacology. 1988; 37(5):837-841

Shenoy AK., Somayaji SN and Bairy KL.Evaluation of hepatoprotective activity of Ginkgobiloba in rats. Indian Journal of Physiology and Pharmacology. 2002; 46(2) :167-174. 
Soundararajan Vijayarathna., Subramanion L Jothy., Kwan Yuet Ping., Lachimanan Yoga Latha., Nadras Othman., and Sreenivasan Sasidharan. In Vitro Antioxidant Activity and Hepatoprotective Potential Of Elaeis Guineensis Leaf Against Paracetamol Induced Damage in Mice. International Journal of Chemical Engineering and Applications. 2012; $3(4)$.

Sreenivasan Sasidharan., Sugumaran Aravindran., Lachimanan Yoga Latha., Ratnasamy Vijenthi., Dharmaraj Saravanan and Santhanam Amutha . In vitro Antioxidant Activity and Hepatoprotective Effects of Lentinula edodes against Paracetamol-Induced Hepatotoxicity. Molecules. 2010; 15: 4478-4489

Vermeulen NPE., Bessems Jam and., Van de Strat R. Molecular aspects of paracetamol induced hepatotoxicity and its mechanism-based prevention. Drug Metab Rev. 1992; 24:367-407.

Yadav SR., Mayur YK and Nilesh VM. A new species of Ceropegia L. (Asclepiadaceae) from the Western Ghats of India with a note on series Attenuatae Huber. Current science. 2006; 91(9):1140-1142.

Yanpallewar SU, Sen S, Tapas S, Kumar M, Raju SS, Acharya SB. Phytomedicine 2002; 9: 391-396.

\section{How to cite this article:}

Kolakota R, Kumar RS, Patnaik SK. In Vitro Antioxidant Activity and Hepatoprotective Potential of Ceropegia spiralis Against Paracetamol Induced liver injury. J App Pharm Sci, 2017; 7 (09): 199-206. 